\title{
A fixed point theorem and the Hyers-Ulam stability in Riesz spaces
}

Bogdan Batko ${ }^{1,2}$ and Janusz Brzdęk ${ }^{1 *}$

${ }^{*}$ Correspondence:

jbrzdek@up.krakow.pl

${ }^{1}$ Department of Mathematics,

Pedagogical University,

Podchorążych 2, Kraków, 30-084, Poland

Full list of author information is

available at the end of the article

\begin{abstract}
We prove a fixed point theorem and show its applications in investigations of the Hyers-Ulam type stability of some functional equations (in single and many variables) in Riesz spaces.

MSC: $39 \mathrm{~B} 82 ; 47 \mathrm{H} 10$

Keywords: Hyers-Ulam type stability; fixed point; Riesz space; square symmetric groupoid
\end{abstract}

\section{Introduction}

The Hyers-Ulam stability for functional, but also for difference, differential and integral equations, is a very quickly growing area of investigations (for more details and further references, see, e.g., [1-7]; examples of some recent results can be found in [8-20]). It is related to the notions of shadowing (see, e.g., [21-23]) as well as to the theories of perturbation (see, e.g., $[24,25]$ ) and optimization. The first known result on such stability is due to Pólya and Szegö [26] and reads as follows.

For every real sequence $\left(a_{n}\right)_{n \in \mathbb{N}}$ with $\sup _{n, m \in \mathbb{N}}\left|a_{n+m}-a_{n}-a_{m}\right| \leq 1$, there is a real number $\omega$ such that $\sup _{n \in \mathbb{N}}\left|a_{n}-\omega n\right| \leq 1$. Moreover, $\omega=\lim _{n \rightarrow \infty} a_{n} / n$.

But it was Ulam ( $c f .[27,28])$ who in 1940 gave the main motivation for the study of that subject, at the University of Wisconsin, where he presented some unsolved problems and in particular the following one.

Let $G_{1}$ be a group and $\left(G_{2}, d\right)$ a metric group. Given $\varepsilon>0$, does there exist $\delta>0$ such that if $f: G_{1} \rightarrow G_{2}$ satisfies $d(f(x y), f(x) f(y))<\delta$ for all $x, y \in G_{1}$, then a homomorphism $T: G_{1} \rightarrow G_{2}$ exists with $d(f(x), T(x))<\varepsilon$ for all $x, y \in G_{1}$ ?

In 1941 Hyers [27] published an answer to it, which is presented below in a bit generalized form (see, e.g., [4]).

Let $(X,+)$ be a commutative semigroup, $Y$ be a Banach space and $\varepsilon>0$. Then for every $g: X \rightarrow Y$ with $\sup _{x, y \in X}\|g(x+y)-g(x)-g(y)\| \leq \varepsilon$ there is a unique $f: X \rightarrow Y$ such that $\sup _{x \in X}\|g(x)-f(x)\| \leq \varepsilon$ and

$$
f(x+y)=f(x)+f(y), \quad x, y \in X .
$$

By now we express that result simply saying that the Cauchy functional equation (1) is Hyers-Ulam stable (or has the Hyers-Ulam stability) in the class of functions $Y^{X}$.

(C) 2013 Batko and Brzdęk; licensee Springer. This is an Open Access article distributed under the terms of the Creative Commons Attribution License (http://creativecommons.org/licenses/by/2.0), which permits unrestricted use, distribution, and reproduction in any medium, provided the original work is properly cited. 
The Hyers-Ulam stability in Riesz spaces have already been studied in [29] (with a direct method) and in [11] (with an application of the spectral representation theorem). The main motivation for this kind of investigations follows from the pretty natural concept to pose the stability problem for a given functional equation in the settings of an ordered structure as an alternative for the topological or metric ones. To preserve the spirit of the HyersUlam stability, we do that in a way which allows to sustain the full coincidence with the classical notion in the simplest case of real functions.

The fixed point approach has been already applied in the investigation of the HyersUlam stability, e.g., in $[8,12,13,30-37]$ and it seems that Baker (see [30]) has used this tool for the first time in this field; for a survey on this subject, see [38]. In this paper we continue this direction following the approach presented in [12,13,31]. We start our paper with a natural fixed point theorem and next derive some stability results from it.

\section{Preliminaries}

In this paper, as usual, $\mathbb{N}, \mathbb{N}_{0}, \mathbb{R}$ and $\mathbb{R}_{+}$denote the sets of all positive integers, nonnegative integers, real numbers and nonnegative real numbers, respectively.

For the readers' convenience, we quote basic definitions and properties concerning Riesz spaces (see [39]).

Definition 1 ( $c f$. [39], Definition 11.1 and Definition 22.1) We say that a real linear space $L$, endowed with a partial order $\leq \subset L^{2}$, is a Riesz space if $\sup \{x, y\}$ exists for all $x, y \in L$ and

$$
a x+y \leq a z+y, \quad x, y, z \in X, x \leq z, a \in \mathbb{R}_{+}
$$

we define the absolute value of $x \in L$ by the formula $|x|:=\sup \{x,-x\} \geq 0$.

A Riesz space $L$ is called Archimedean if, for each $x \in L$, the inequality $x \leq 0$ holds whenever the set $\{n x: n \in \mathbb{N}\}$ is bounded from above.

It is easily seen that in a Riesz space $L$ we have $|v| \geq 0$ and $\alpha u \leq \beta u$ for every $v, u \in L$, $u \geq 0$, and $\alpha, \beta \in \mathbb{R}, \alpha \leq \beta$.

There are several types of convergence that may be defined according to the order structure. One of them is the relatively uniform convergence defined as follows.

Definition 2 (cf. [39], Definition 39.1) Let $L$ be a Riesz space and $e \in L$. A sequence $\left\{f_{n}\right\}_{n \in \mathbb{N}}$ in $L$ is said to converge e-uniformly to an element $f \in L$ whenever, for every $\varepsilon>0$, there exists a positive integer $n_{0}$ such that $\left|f-f_{n}\right| \leq \varepsilon e$ holds for all $n \geq n_{0}$. A sequence $\left\{f_{n}\right\}_{n \in \mathbb{N}}$ in $L$ is called e-uniform Cauchy sequence whenever, for every $\varepsilon>0$, there exists a positive integer $n_{1}$ such that $\left|f_{m}-f_{n}\right| \leq \varepsilon e$ holds for all $m, n \geq n_{1}$.

Let us point out (see $[39, \mathrm{p} .252]$ ) that in a Riesz space $L$ that is Archimedean, the $e$ uniform limit of a sequence in it, if exists, is unique and the fact that $\left\{f_{n}\right\}_{n \in \mathbb{N}}$ converges $e$-uniformly to $f$ will be denoted by $\lim _{n \rightarrow \infty}^{e} f_{n}=f$ (in particular, if $v \in L$ and $f_{n} \geq v$ for $n \in \mathbb{N}$, then $f \geq v$ ).

Definition 3 The series $\sum_{i=0}^{\infty} a_{i}$ in a Riesz space $L$ is said to converge e-uniformly (for a given $e \in L$ ) to an element $S \in L$ whenever the sequence $S_{n}:=\sum_{i=0}^{n} a_{i}$ converges $e$ uniformly to $S$. If $L$ is Archimedean, then we write $\sum_{i=0}^{\infty} a_{i} \stackrel{e}{=} S$. 
Definition 4 (cf. [39], Definition 39.3) A Riesz space $L$ is called e-uniformly complete (with a given $e \in L$ ) whenever every $e$-uniform Cauchy sequence has an $e$-uniform limit.

There is a large class of spaces satisfying the above conditions. In particular, every Dedekind $\sigma$-complete space (that is such that any non-empty at most countable subset which is bounded from above has a supremum) is an Archimedean $e$-uniformly complete space for every $e \geq 0$ (see [39, pp.125, 252, 253]).

\section{Main result}

In what follows $X$ is a nonempty set and $Y$ is an Archimedean $u$-uniformly complete Riesz space for some given $u \in Y$.

We start that part with a lemma that is quite elementary. However, since we use it several times, we present it with a proof for the convenience of readers.

Lemma 1 Let $L$ be an Archimedean Riesz space and $e \in L$. The sequence of rests $r_{n}:=$ $\sum_{i=n+1}^{\infty} a_{i}$ of an e-uniformly convergent series $\sum_{i=0}^{\infty} a_{i}$ in $L$ is e-uniformly convergent to 0.

Proof Let us observe that $\left|\sum_{i=n+1}^{m} a_{i}-S+S_{n}\right|=\left|S_{m}-S_{n}-S+S_{n}\right|=\left|S_{m}-S\right|$. Consequently, $\sum_{i=n+1}^{\infty} a_{i} \stackrel{e}{=} S-S_{n} \in L$ as $S_{m}$ converges $e$-uniformly to $S$ and, therefore, $r_{n}$ is well defined. Moreover, we have $\lim _{n \rightarrow \infty}^{e} r_{n}=\lim _{n \rightarrow \infty}^{e}\left(S-S_{n}\right)=0 \in L$.

The following two hypotheses will be useful.

(H1) Mappings $f_{1}, \ldots, f_{k}: X \rightarrow X$ and $L_{1}, \ldots, L_{k}: X \rightarrow \mathbb{R}_{+}$are given and $\mathcal{J}: Y^{X} \rightarrow Y^{X}$ is an operator satisfying the inequality

$$
|(\mathcal{J} a)(t)-(\mathcal{J} b)(t)| \leq \sum_{i=1}^{k} L_{i}(t)\left|a\left(f_{i}(t)\right)-b\left(f_{i}(t)\right)\right|, \quad a, b \in Y^{X}, t \in X
$$

(H2) $\Lambda: Y^{X} \rightarrow Y^{X}$ is a linear operator given by

$$
(\Lambda \delta)(t):=\sum_{i=1}^{k} L_{i}(t) \delta\left(f_{i}(t)\right), \quad \delta \in Y^{X}, t \in X
$$

Observe that $\Lambda$ is monotone with respect to the pointwise ordering (because $L_{i}$ is nonnegative). Our main theorem reads as follows.

Theorem 1 Let us assume that hypotheses $(\mathrm{H} 1)$ and $(\mathrm{H} 2)$ are fulfilled and that functions $\varphi, \varepsilon, S: X \rightarrow Y$ satisfy the conditions

$$
\begin{aligned}
& |(\mathcal{J} \varphi)(t)-\varphi(t)| \leq \varepsilon(t), \quad t \in X, \\
& \sum_{n=0}^{\infty}\left(\Lambda^{n} \varepsilon\right)(t) \stackrel{u}{=} S(t), \quad t \in X .
\end{aligned}
$$

Then $\mathcal{J}$ has a unique fixed point $\psi \in Y^{X}$ with

$$
|\varphi(t)-\psi(t)| \leq S(t), \quad t \in X
$$


Moreover,

$$
\psi(t)=\lim _{n \rightarrow \infty} u\left(\mathcal{J}^{n} \varphi\right)(t), \quad t \in X .
$$

Proof The proof is somewhat similar to the proof of [12, Theorem 1], but since there are also significant differences between them, we present it here.

First we show by induction that

$$
\left|\left(\mathcal{J}^{n} \varphi\right)(t)-\left(\mathcal{J}^{n+1} \varphi\right)(t)\right| \leq\left(\Lambda^{n} \varepsilon\right)(t), \quad t \in X
$$

For $n=0,(8)$ and (4) coincide. So, let us suppose that (8) holds for a fixed $n \in \mathbb{N}$. Using (2) and the definition of $\Lambda$ (i.e., (3)), we have

$$
\begin{aligned}
& \left|\left(\mathcal{J}^{n+1} \varphi\right)(t)-\left(\mathcal{J}^{n+2} \varphi\right)(t)\right| \\
& \leq \sum_{i=1}^{k} L_{i}(t)\left|\left(\mathcal{J}^{n} \varphi\right)\left(f_{i}(t)\right)-\left(\mathcal{J}^{n+1} \varphi\right)\left(f_{i}(t)\right)\right| \\
& \leq \sum_{i=1}^{k} L_{i}(t)\left(\Lambda^{n} \varepsilon\right)\left(f_{i}(t)\right) \\
& =\left(\Lambda^{n+1} \varepsilon\right)(t), \quad t \in X,
\end{aligned}
$$

which completes the proof of (8). Therefore, for $n, k \in \mathbb{N}$, we get

$$
\left|\left(\mathcal{J}^{n} \varphi\right)(t)-\left(\mathcal{J}^{n+k} \varphi\right)(t)\right| \leq \sum_{i=n}^{n+k-1}\left(\Lambda^{i} \varepsilon\right)(t), \quad t \in X .
$$

This, according to (5) and Lemma 1, means that $\left((\mathcal{J} \varphi)^{n}(t)\right)_{n \in \mathbb{N}}$ is a $u$-uniform Cauchy sequence for any $t \in X$, and therefore $u$-uniformly convergent, as $Y$ is $u$-uniformly complete. Let us denote by $\psi(t)$ its relative uniform limit. Using (9) with $n=0$, we have

$$
\left|\varphi(t)-\left(\mathcal{J}^{k} \varphi\right)(t)\right| \leq \sum_{i=0}^{k-1}\left(\Lambda^{i} \varepsilon\right)(t), \quad t \in X .
$$

Write $S_{k-1}(t):=\sum_{i=0}^{k-1}\left(\Lambda^{i} \varepsilon\right)(t)$ for $k \in \mathbb{N}$ and $t \in X$. By the triangle inequality and (10), for any $k \in \mathbb{N}$ and $t \in X$, we obtain

$$
\begin{aligned}
|\varphi(t)-\psi(t)| & \leq\left|\varphi(t)-\left(\mathcal{J}^{k} \varphi\right)(t)\right|+\left|\left(\mathcal{J}^{k} \varphi\right)(t)-\psi(t)\right| \\
& \leq S_{k-1}(t)+\left|\left(\mathcal{J}^{k} \varphi\right)(t)-\psi(t)\right| \\
& \leq\left|S_{k-1}(t)-S(t)\right|+\left|\left(\mathcal{J}^{k} \varphi\right)(t)-\psi(t)\right|+S(t) .
\end{aligned}
$$

From the $u$-uniform convergence of $\left((\mathcal{J} \varphi)^{k}(t)\right)_{k \in \mathbb{N}}$ to $\psi(t)$ and $\left(S_{k-1}(t)\right)_{k \in \mathbb{N}}$ to $S(t)$, for $t \in X$, we derive

$$
|\varphi(t)-\psi(t)| \leq S(t)+\frac{1}{n} u, \quad n \in \mathbb{N}, t \in X,
$$

which results in (6) as $Y$ is Archimedean. 
We will show that $\psi$ is a fixed point of $\mathcal{J}$. Having applied (2) with $a=\psi$ and $b=\mathcal{J}^{n-1} \varphi$, for any $t \in X$, one has

$$
\left|(\mathcal{J} \psi)(t)-\mathcal{J}\left(\mathcal{J}^{n-1} \varphi\right)(t)\right| \leq \sum_{i=1}^{k} L_{i}(t)\left|\psi\left(f_{i}(t)\right)-\mathcal{J}^{n-1} \varphi\left(f_{i}(t)\right)\right|, \quad n \in \mathbb{N} .
$$

Next, using the triangle inequality $|(\mathcal{J} \psi)(t)-\psi(t)| \leq\left|(\mathcal{J} \psi)(t)-\left(\mathcal{J}^{n} \varphi\right)(t)\right|+\mid\left(\mathcal{J}^{n} \varphi\right)(t)-$ $\psi(t) \mid$ (for $n \in \mathbb{N}, t \in X$ ), we infer that

$$
|(\mathcal{J} \psi)(t)-\psi(t)| \leq \frac{1}{m} u\left(\sum_{i=1}^{k} L_{i}(t)+1\right), \quad t \in X, m \in \mathbb{N}
$$

which means that $\mathcal{J} \psi=\psi$ as $Y$ is Archimedean.

For the proof of the uniqueness of $\psi$, assume that $\psi_{1}, \psi_{2} \in Y^{X}$ are two fixed points of $\mathcal{J}$ satisfying

$$
\left|\varphi(t)-\psi_{i}(t)\right| \leq S(t), \quad t \in X, i=1,2 .
$$

At first let us observe that for every $n \in \mathbb{N}$, we get

$$
\left|\left(\mathcal{J}^{n} \psi_{1}\right)(t)-\left(\mathcal{J}^{n} \psi_{2}\right)(t)\right| \leq 2 \sum^{\infty}\left(\Lambda^{i} \varepsilon\right)(t), \quad t \in X
$$

In fact, for $n=0$ inequality (15) follows directly from (14). Now assume that (15) holds for a given $n \in \mathbb{N}$. Then, by (12), for every $t \in X$, we have

$$
\begin{aligned}
& \left|\left(\mathcal{J}^{n+1} \psi_{1}\right)(t)-\left(\mathcal{J}^{n+1} \psi_{2}\right)(t)\right| \\
& \quad=\left|\left(\mathcal{J}\left(\mathcal{J}^{n} \psi_{1}\right)\right)(t)-\left(\mathcal{J}\left(\mathcal{J}^{n} \psi_{2}\right)\right)(t)\right| \\
& \quad \leq \sum_{i=1}^{k} L_{i}(t)\left|\left(\mathcal{J}^{n} \psi_{1}\right)\left(f_{i}(t)\right)-\left(\mathcal{J}^{n} \psi_{2}\right)\left(f_{i}(t)\right)\right| \\
& \quad \leq 2 \sum_{i=1}^{k} L_{i}(t) \sum_{j=n}^{\infty}\left(\Lambda^{j} \varepsilon\right)\left(f_{i}(t)\right) \\
& \quad=2 \sum_{j=n}^{\infty} \sum_{i=1}^{k} L_{i}(t)\left(\Lambda^{j} \varepsilon\right)\left(f_{i}(t)\right) \\
& \quad=2 \sum_{j=n+1}^{\infty}\left(\Lambda^{j} \varepsilon\right)(t) .
\end{aligned}
$$

Thus we have proved (15). Letting $n \rightarrow \infty$ and taking into account Lemma 1, we infer that $\psi_{1}=\psi_{2}$.

Remark 1 If in (2) we take $k=1, f_{1}(x)=x$ and $L_{1}(x)=\lambda \in[0,1)$ for $x \in X$, then Theorem 1 becomes a result corresponding to the classical Banach contraction principle. 
Remark 2 We can associate to a given $u \in Y$ an extended (i.e., admitting the infinite value) norm on $Y$ denoted by $\|\cdot\|_{u}$ and defined by

$$
\|v\|_{u}:=\inf \left\{\lambda \in \mathbb{R}_{+}:|v| \leq \lambda u\right\}, \quad v \in Y .
$$

From Theorem 1 we can easily deduce the following corollary.

Corollary 1 Let hypothesis (H1) be fulfilled, $\Lambda_{0}: \mathbb{R}_{+}^{X} \rightarrow \mathbb{R}_{+}^{X}$ be a linear operator given by

$$
\left(\Lambda_{0} \delta\right)(t):=\sum_{i=1}^{k} L_{i}(t) \delta\left(f_{i}(t)\right), \quad \delta \in \mathbb{R}_{+}^{X}, t \in X,
$$

and functions $\varphi: X \rightarrow Y$ and $\varepsilon_{0}: X \rightarrow \mathbb{R}_{+}$satisfy the conditions

$$
\begin{gathered}
\|(\mathcal{J} \varphi)(t)-\varphi(t)\|_{u} \leq \varepsilon_{0}(t), \quad t \in X, \\
S_{0}(t):=\sum_{n=0}^{\infty}\left(\Lambda_{0}^{n} \varepsilon_{0}\right)(t)<\infty, \quad t \in X .
\end{gathered}
$$

Then $\mathcal{J}$ has a unique fixed point $\psi \in Y^{X}$ with

$$
\|\varphi(t)-\psi(t)\|_{u} \leq S_{0}(t), \quad t \in X
$$

Moreover, $\psi$ is given by (7).

Proof Define $S, \varepsilon: X \rightarrow Y$ by $S(t):=S_{0}(t) u$ and $\varepsilon(t):=\varepsilon_{0}(t) u$ for $t \in X$. Next let $\Lambda: Y^{X} \rightarrow$ $Y^{X}$ be given by (3). Then it is easily seen that (4) and (5) are valid. Consequently, in view of Theorem 1, $\mathcal{J}$ has a unique fixed point $\psi \in Y^{X}$ such that (6) holds; moreover, $\psi$ is given by (7). Clearly, (6) and (19) are equivalent, whence we derive the statement concerning the uniqueness of $\psi$.

\section{The Hyers-Ulam stability}

In the simplest case $k=1$, Theorem 1 yields at once the following corollary concerning the Hyers-Ulam stability of a quite general functional equation

$$
\psi(t)=F(t, \psi(\xi(t)))
$$

in the class of functions $Y^{X}$, with given $F: X \times Y \rightarrow Y$ and $\xi: X \rightarrow X$.

Corollary 2 Let $F: X \times Y \rightarrow Y, \xi: X \rightarrow X, \lambda: X \rightarrow \mathbb{R}_{+}, \varphi, \varepsilon, S: X \rightarrow Y$,

$$
\begin{aligned}
& |F(t, u)-F(t, v)| \leq \lambda(t)|u-v|, \quad t \in X, u, v \in Y, \\
& S(t) \stackrel{u}{=} \varepsilon(t)+\sum_{n=0}^{\infty}\left(\prod_{i=0}^{n} \lambda\left(\xi^{i}(t)\right)\right) \varepsilon\left(\xi^{n+1}(t)\right), \quad t \in X,
\end{aligned}
$$

and

$$
|\varphi(t)-F(t, \varphi(\xi(t)))| \leq \varepsilon(t), \quad t \in X
$$


Then there is a unique solution $\psi \in Y^{X}$ of functional equation (20) such that

$$
|\psi(t)-\varphi(t)| \leq S(t), \quad t \in X
$$

Moreover, $\psi$ is given by $(7)$ with $(\mathcal{T} g)(t)=F(t, g(\xi(t)))$ for $g \in Y^{X}, t \in X$.

Proof Use Theorem 1 with $k=1, f_{1}=\xi, L_{1}=\lambda$, and $(\mathcal{J} g)(t)=F(t, g(\xi(t)))$ for $g \in Y^{X}$, $t \in X$.

Example 1 If there is $\alpha \in \mathbb{R}_{+}$such that (in Corollary 2) $\lambda(t) \leq \alpha$ for $t \in X$, then clearly we get $S(t) \leq \sum_{n=0}^{\infty} \alpha^{n} \varepsilon\left(\xi^{n}(t)\right)$ for $t \in X$, provided $\sum_{n=0}^{\infty} \alpha^{n} \varepsilon\left(\xi^{n}(t)\right)$ is $u$-uniformly convergent in $Y$. Moreover, in the case where $\varepsilon(f(t)) \leq \beta \varepsilon(t)$ for all $t \in X$ and some $\beta \in \mathbb{R}_{+}$with $\alpha \beta<1$ (e.g., when $\alpha<1$ and $\varepsilon$ is a constant function), $S(t) \leq \frac{1}{1-\alpha \beta} \varepsilon(t)$ for $t \in S$. This means that Corollary 2 implies some analogues of the results in [30] for the Riesz spaces.

Analogously, from Corollary 1 we obtain the following.

Corollary 3 Let $F: X \times Y \rightarrow Y, \xi: X \rightarrow X, \varepsilon_{0}, \lambda: X \rightarrow \mathbb{R}_{+}, \varphi: X \rightarrow Y,(21)$ holds,

$$
\begin{aligned}
& S_{0}(t):=\varepsilon_{0}(t)+\sum_{n=0}^{\infty}\left(\prod_{i=0}^{n} \lambda\left(\xi^{i}(t)\right)\right) \varepsilon_{0}\left(\xi^{n+1}(t)\right)<\infty, \quad t \in X, \\
& \|\varphi(t)-F(t, \varphi(\xi(t)))\|_{u} \leq \varepsilon_{0}(t), \quad t \in X .
\end{aligned}
$$

Then there is a unique solution $\psi \in Y^{X}$ of equation (20) such that

$$
\|\psi(t)-\varphi(t)\|_{u} \leq S_{0}(t), \quad t \in X
$$

Moreover, $\psi$ is given by (7).

Example 2 If (in Corollaries 2 and 3) $F(t, v)=b(t) v+H(t)$ for $t \in X, v \in Y$ with some $b: X \rightarrow \mathbb{R}, \xi: X \rightarrow X$ and $H: X \rightarrow Y$, then we get stability results for the equation

$$
\Phi(t)=b(t) \Phi(\xi(t))+H(t)
$$

corresponding to [40, Theorem 2.1 and Corollary 2.4], from which we can easily derive stability results analogous to [41, Theorem 2.1] (provided $0 \notin b(X)$ ) for the equation

$$
\Phi(\xi(t))=\frac{1}{b(t)} \Phi(t)-\frac{H(t)}{b(t)} .
$$

We also can use Theorem 1 and Corollary 1 to deal with the stability of a bit more involved equations with any number of unknown functions, e.g., as follows.

Corollary 4 Let $k, m \in \mathbb{N}, L_{1}, \ldots, L_{k}: X \rightarrow \mathbb{R}_{+}, G: X \times Y^{m} \rightarrow Y, f_{1}, \ldots, f_{k}, g_{1}, \ldots, g_{m}: X \rightarrow$ $X, \Phi: X \times Y^{k} \rightarrow Y$ and

$$
\left|\Phi\left(t, u_{1}, \ldots, u_{k}\right)-\Phi\left(t, v_{1}, \ldots, v_{k}\right)\right| \leq \sum_{i=1}^{k} L_{i}(t)\left|u_{i}-v_{i}\right|, \quad u_{i}, v_{i} \in Y, t \in X
$$


Suppose that functions $\varphi, \varepsilon, \mu_{1}, \ldots, \mu_{m}: X \rightarrow Y$ satisfy the inequality

$$
\left|\varphi(t)-\Phi\left(t, \varphi\left(f_{1}(t)\right), \ldots, \varphi\left(f_{k}(t)\right)\right)-G\left(t, \mu_{1}\left(g_{1}(t)\right), \ldots, \mu_{m}\left(g_{m}(t)\right)\right)\right| \leq \varepsilon(t), \quad t \in X,
$$

and (5) holds with $\Lambda$ given by (3). Then there exists a unique $\psi: X \rightarrow Y$ satisfying inequality (24), which solves the equation

$$
\psi(t)=\Phi\left(t, \psi\left(f_{1}(t)\right), \ldots, \psi\left(f_{k}(t)\right)\right)+G\left(t, \mu_{1}\left(g_{1}(t)\right), \ldots, \mu_{m}\left(g_{m}(t)\right)\right) .
$$

Moreover,

$$
\psi(t)=\lim _{n \rightarrow \infty} u\left(T_{0}^{n} \varphi\right)(t), \quad t \in X,
$$

where $\left(T_{0} h\right)(t):=\Phi\left(t, h\left(f_{1}(t)\right), \ldots, h\left(f_{k}(t)\right)\right)+G\left(t, \mu_{1}\left(g_{1}(t)\right), \ldots, \mu_{m}\left(g_{m}(t)\right)\right)$ for $h \in Y^{X}, t \in X$.

Proof It is enough to use Theorem 1 with $\mathcal{J}=T_{0}$.

Corollary 5 Let $k, m \in \mathbb{N}, L_{1}, \ldots, L_{k}: X \rightarrow \mathbb{R}_{+}, G: X \times Y^{m} \rightarrow Y, f_{1}, \ldots, f_{k}, g_{1}, \ldots, g_{m}: X \rightarrow$ $X$, and $\Phi: X \times Y^{k} \rightarrow Y$ satisfy (28). Suppose that functions $\varphi, \mu_{1}, \ldots, \mu_{m}: X \rightarrow Y$ and $\varepsilon: X \rightarrow \mathbb{R}_{+}$are such that

$$
\left\|\varphi(t)-\Phi\left(t, \varphi\left(f_{1}(t)\right), \ldots, \varphi\left(f_{k}(t)\right)\right)-G\left(t, \mu_{1}\left(g_{1}(t)\right), \ldots, \mu_{m}\left(g_{m}(t)\right)\right)\right\|_{u} \leq \varepsilon(t), \quad t \in X,
$$

and (18) holds with $\Lambda_{0}$ given by (16). Then there exists a unique $\psi: X \rightarrow Y$ satisfying inequality (27) and equation (29). Moreover, $\psi$ is given by (30).

Proof It is enough to use Corollary 1 with $\mathcal{J}=T_{0}$.

In the next part we supply an example of a result concerning the Hyers-Ulam stability of functional equations in several variables.

\section{Stability of the equation of homomorphism for the square symmetric groupoids}

Let $T$ be a nonempty set. A binary operation $\star: T^{2} \rightarrow T$ is square symmetric provided

$$
(x \star y) \star(x \star y)=(x \star x) \star(y \star y), \quad x, y \in T
$$

(see, e.g., $[18,42-45])$. If $(T,+)$ is a commutative semigroup, $\gamma_{0} \in T$ and $\alpha, \beta: T \rightarrow T$ are endomorphisms with $\alpha \circ \beta=\beta \circ \alpha$ (for instance, $\alpha=\beta^{n}$ for an $n \in \mathbb{N}_{0}$ ), then it is easy to check that $*: T^{2} \rightarrow T$, given by

$$
x * y:=\alpha(x)+\beta(y)+\gamma_{0}, \quad x, y \in T,
$$

is square symmetric. So, it is easily seen that the following two very well-known functional equations

$$
\begin{aligned}
& f(x+y)=f(x)+f(y) \quad \text { (Cauchy), } \\
& f\left(\frac{x+y}{2}\right)=\frac{f(x)+f(y)}{2} \quad \text { (Jensen) }
\end{aligned}
$$


are particular examples of homomorphism equations for square symmetric groupoids. Moreover, we have a very particular case of a square symmetric operation when $T$ is a linear space over a field $\mathbb{K}$ and $*$ is defined by

$$
s * t:=C s+D t+u, \quad s, t \in T,
$$

with some fixed $C, D \in \mathbb{K}$ and $u \in T$. So, the general linear functional equation

$$
f(C x+D y+u)=A f(x)+B f(y)+c
$$

(for instance, with some fixed $A, B \in \mathbb{K}, c \in T$ and for functions $f \in T^{T}$ ) is also an example of a homomorphism equation for some square symmetric groupoids.

The next corollary provides an example of stability result for the functional equation of homomorphism for square symmetric groupoids, which corresponds to some outcomes in $[18,42-44]$.

Corollary 6 Let $C, D \in \mathbb{R}, C \neq-D, S, \varphi: X \rightarrow Y, \chi: X^{2} \rightarrow Y, w \in Y, \star: X^{2} \rightarrow X$ be a square symmetric binary operation, and let $\rho: X \rightarrow X$ be given by $\rho(t):=t \star t$ for $t \in X$,

$$
\begin{aligned}
& |\varphi(s \star t)-C \varphi(s)-D \varphi(t)-w| \leq \chi(s, t), \quad s, t \in X, \\
& S(t) \stackrel{u}{=} \sum_{n=0}^{\infty}|C+D|^{-n-1} \chi\left(\rho^{n}(t), \rho^{n}(t)\right), \quad t \in X,
\end{aligned}
$$

and

$$
\lim _{n \rightarrow \infty}{ }^{u}|C+D|^{-n} \chi\left(\rho^{n}(t), \rho^{n}(s)\right)=0, \quad s, t \in X
$$

Then there exists a unique solution $\Phi \in Y^{X}$ of the functional equation

$$
\Phi(s \star t)=C \Phi(s)+D \Phi(t)+w
$$

such that

$$
|\varphi(t)-\Phi(t)| \leq S(t), \quad t \in X
$$

Moreover,

$$
\Phi(t)=\lim _{n \rightarrow \infty} u\left[(C+D)^{-n} \varphi\left(\rho^{n}(t)\right)-\sum_{i=1}^{n}(C+D)^{-i} w\right], \quad t \in X .
$$

Proof Take $s=t$ in (32). Then we get

$$
|\varphi(\rho(s))-(C+D) \varphi(s)-w| \leq \chi(s, s), \quad s \in X,
$$

and consequently

$$
\left|(C+D)^{-1} \varphi(\rho(s))-(C+D)^{-1} w-\varphi(s)\right| \leq|C+D|^{-1} \chi(s, s), \quad s \in X .
$$


Hence (21) and (23) hold with $\xi=\rho$ and

$$
F(t, v):=(C+D)^{-1}(v-w), \quad \varepsilon(t):=|C+D|^{-1} \chi(t, t), \quad \lambda(t):=|C+D|^{-1}
$$

for $v \in Y, t \in X$. Now, if $S: X \rightarrow Y$ is defined by (22), then it is easily seen that (33) holds. Consequently, according to Corollary 2, there exists a unique solution $\Phi \in Y^{X}$ of the equation

$$
\varphi(\rho(s))=(C+D) \varphi(s)+w
$$

such that condition (36) is fulfilled. Moreover, $\Phi$ is given by (37).

Now we show that $\Phi$ is a solution of equation (35). To this end, fix $s, t \in X$ and $n \in \mathbb{N}_{0}$. Then $\rho^{n}(s \star t)=\rho^{n}(s) \star \rho^{n}(t)$, whence by (32),

$$
\begin{aligned}
\left|C \mathcal{T}^{n} \varphi(s)+D \mathcal{T}^{n} \varphi(t)+w-\mathcal{T}^{n} \varphi(s \star t)\right| \\
=\mid C\left((C+D)^{-n} \varphi\left(\rho^{n}(s)\right)-\sum_{i=1}^{n}(C+D)^{-i} w\right) \\
\quad+D\left((C+D)^{-n} \varphi\left(\rho^{n}(t)\right)-\sum_{i=1}^{n}(C+D)^{-i} w\right)+w \\
\quad-\left((C+D)^{-n} \varphi\left(\rho^{n}(s \star t)\right)-\sum_{i=1}^{n}(C+D)^{-i} w\right) \mid \\
\leq|C+D|^{-n}\left|C \varphi\left(\rho^{n}(s)\right)+D \varphi\left(\rho^{n}(t)\right)+w-\varphi\left(\rho^{n}(s) \star \rho^{n}(t)\right)\right| \\
\leq|C+D|^{-n} \chi\left(\rho^{n}(s), \rho^{n}(t)\right) .
\end{aligned}
$$

This means that $\Phi$ satisfies (35) (in view of (34) and (37)).

Since $\Phi \in X^{S}$ is the unique solution of functional equation (41) fulfilling (36), and every solution of (35) satisfies (41), we also obtain the statement concerning the uniqueness of $\Phi$.

Corollary 7 Let $C, D \in \mathbb{R}, C \neq-D, \varphi: X \rightarrow Y, \chi_{0}: X^{2} \rightarrow \mathbb{R}_{+}, w \in Y, \star: X^{2} \rightarrow X$ be a square symmetric binary operation, let $\rho: X \rightarrow X$ be given by $\rho(t):=t \star t$ for $t \in X$,

$$
\begin{aligned}
& \|\varphi(s \star t)-C \varphi(s)-D \varphi(t)-w\|_{u} \leq \chi_{0}(s, t), \quad s, t \in X, \\
& S_{0}(t):=\sum_{n=0}^{\infty} \frac{\chi_{0}\left(\rho^{n}(t), \rho^{n}(t)\right)}{|C+D|^{n+1}}<\infty, \quad t \in X
\end{aligned}
$$

and

$$
\lim _{n \rightarrow \infty} \frac{\chi_{0}\left(\rho^{n}(s), \rho^{n}(t)\right)}{|C+D|^{n}}=0, \quad s, t \in X
$$

Then there is a unique solution $\Phi \in Y^{X}$ of equation (35) such that

$$
\|\varphi(t)-\Phi(t)\|_{u} \leq S_{0}(t), \quad t \in X .
$$

Moreover, $\Phi$ is given by (37). 
Proof We argue as in the proof of Corollary 1. Namely, define $S: X \rightarrow Y$ and $\chi: X^{2} \rightarrow Y$ by $S(t):=S_{0}(t) u$ and $\chi(t):=\chi_{0}(t) u$ for $t \in X$. Then (42)-(44) imply (32)-(34). Hence, by Corollary 6, there exists a unique solution $\Phi \in Y^{X}$ of functional equation (35) such that (36) holds; in particular, $\Phi$ is given by (37). Clearly (36) and (45) are equivalent, so we also obtain the statement on the uniqueness of $\Phi$.

In the very particular case where $C=D=1$ and functions $\chi$ and $\chi_{0}$ are constant, Corollaries 6 and 7 yield analogues of the classical result of Hyers [27].

Competing interests

The authors declare that they have no competing interests.

Authors' contributions

All authors contributed equally to the manuscript. All authors have read and approved the final version of the manuscript.

\section{Author details}

${ }^{1}$ Department of Mathematics, Pedagogical University, Podchorążych 2, Kraków, 30-084, Poland. ²Department of Mathematics, WSB - NLU, Zielona 27, Nowy Sącz, 33-300, Poland

Received: 8 January 2013 Accepted: 23 April 2013 Published: 14 May 2013

References

1. Agarwal, RP, Xu, B, Zhang, W: Stability of functional equations in single variable. J. Math. Anal. Appl. 288, $852-869$ (2003)

2. Brillouët-Belluot, N, Brzdęk, J, Ciepliński, K: On some recent developments in Ulam's type stability. Abstr. Appl. Anal. 2012, Article ID 716936 (2012)

3. Czerwik, S: Functional Equations and Inequalities in Several Variables. World Scientific, River Edge (2002)

4. Hyers, DH, Isac, G, Rassias, TM: Stability of Functional Equations in Several Variables. Progress in Nonlinear Differential Equations and Their Applications, vol. 34. Birkhäuser, Boston (1998)

5. Jung, S-M: Hyers-Ulam-Rassias Stability of Functional Equations in Mathematical Analysis. Hadronic Press, Palm Harbor (2001)

6. Jung, S-M: Hyers-Ulam-Rassias Stability of Functional Equations in Nonlinear Analysis. Springer, New York (2011)

7. Moszner, Z: On the stability of functional equations. Aequ. Math. 77, 33-88 (2009)

8. Badora, R, Brzdęk, J: A note on a fixed point theorem and the Hyers-Ulam stability. J. Differ. Equ. Appl. 18, 1115-1119 (2012)

9. Batko, B: On the stability of an alternative functional equation. Math. Inequal. Appl. 8, 685-691 (2005)

10. Batko, B: Stability of an alternative functional equation. J. Math. Anal. Appl. 339, 303-311 (2008)

11. Batko, B, Tabor, J: Stability of the generalized alternative Cauchy equation. Abh. Math. Semin. Univ. Hamb. 69, 67-73 (1999)

12. Brzdęk, J, Chudziak, J, Palés, Z: A fixed point approach to stability of functional equations. Nonlinear Anal. 74, 6728-6732 (2011)

13. Brzdęk, J, Ciepliński, K: A fixed point approach to the stability of functional equations in non-Archimedean metric spaces. Nonlinear Anal. 74, 6861-6867 (2011)

14. Forti, G-L: Comments on the core of the direct method for proving Hyers-Ulam stability of functional equations. J. Math. Anal. Appl. 295, 127-133 (2004)

15. Forti, G-L: Elementary remarks on Ulam-Hyers stability of linear functional equations. J. Math. Anal. Appl. 328, 109-118 (2007)

16. Forti, G-L, Sikorska, J: Variations on the Drygas equation and its stability. Nonlinear Anal. 74, 343-350 (2011)

17. Jabłoński, W, Reich, L: Stability of the translation equation in rings of formal power series and partial extensibility of one-parameter groups of truncated formal power series. Osterreich. Akad. Wiss. Math.-Natur. KI. Sitzungsber. II 215, 127-137 (2006).

18. Páles, Z: Hyers-Ulam stability of the Cauchy functional equation on square-symmetric groupoids. Publ. Math. (Debr.) 58, 651-666 (2001)

19. Popa, D: Hyers-Ulam-Rassias stability of a linear recurrence. J. Math. Anal. Appl. 309, 591-597 (2005)

20. Sikorska, J: On a direct method for proving the Hyers-Ulam stability of functional equations. J. Math. Anal. Appl. 372 , 99-109 (2010)

21. Hayes, W, Jackson, KR: A survey of shadowing methods for numerical solutions of ordinary differential equations. Appl. Numer. Math. 53, 299-321 (2005)

22. Palmer, K: Shadowing in Dynamical Systems. Theory and Applications. Mathematics and Its Applications, vol. 501 Kluwer Academic, Dordrecht (2000)

23. Pilyugin, SY: Shadowing in Dynamical Systems. Lectures Notes in Mathematics, vol. 1706. Springer, Berlin (1999)

24. Chang, KW, Howes, FA: Nonlinear Singular Perturbation Phenomena: Theory and Application. Springer, Berlin (1984)

25. Lin, Z-C, Zhou, M-R: Perturbation Methods in Applied Mathematics. Jiangsu Education Press, Nanjing (1995)

26. Pólya, G, Szegö, G: Aufgaben und Lehrsätze aus der Analysis I. Springer, Berlin (1925)

27. Hyers, DH: On the stability of the linear functional equation. Proc. Natl. Acad. Sci. USA 27, $222-224$ (1941)

28. Ulam, SM: Problems in Modern Mathematics, science ed., Wiley, New York (1964)

29. Polat, F: Approximate Riesz algebra-valued derivations. Abstr. Appl. Anal. 2012, Article ID 240258 (2012) 
30. Baker, JA: The stability of certain functional equations. Proc. Am. Math. Soc. 112, 729-732 (1991)

31. Cădariu, L, Găvruţa, L, Găvruţa, P: Fixed points and generalized Hyers-Ulam stability. Abstr. Appl. Anal. 2012, Article ID 712743 (2012). doi:10.1155/2012/712743

32. Cădariu, L, Radu, V: Fixed point methods for the generalized stability of functional equations in a single variable. Fixed Point Theory Appl. 2008, Article ID 749392 (2008). doi:10.1155/2008/749392

33. Găvruţa, P, Găvruţa, L: A new method for the generalized Hyers-Ulam-Rassias stability. Int. J. Nonlinear Anal. Appl. 1(2), $11-18(2010)$

34. Jung, S-M: A fixed point approach to the stability of isometries. J. Math. Anal. Appl. 329, 879-890 (2007)

35. Jung, S-M, Kim, T-S: A fixed point approach to the stability of the cubic functional equation. Bol. Soc. Mat. Mexicana $12,51-57(2006)$

36. Jung, Y-S, Chang, I-S: The stability of a cubic type functional equation with the fixed point alternative. J. Math. Anal. Appl. 306, 752-760 (2005)

37. Mirzavaziri, M, Moslehian, MS: A fixed point approach to stability of a quadratic equation. Bull. Braz. Math. Soc. 37, 361-376 (2006)

38. Ciepliński, K: Applications of fixed point theorems to the Hyers-Ulam stability of functional equations - a survey. Ann. Funct. Anal. 3, 151-164 (2012)

39. Luxemburg, WAJ, Zaanen, AC: Riesz Spaces. North-Holland, Amsterdam (1971)

40. Brzdęk, J, Popa, D, Xu, B: Selections of set-valued maps satisfying a linear inclusion in a single variable. Nonlinear Anal. 74, 324-330 (2011)

41. Trif, T: On the stability of a general gamma-type functional equation. Publ. Math. (Debr.) 60, 47-61 (2002)

42. Kim, GH: On the stability of functional equations with square-symmetric operation. Math. Inequal. Appl. 4, 257-266 (2001)

43. Kim, GH: Addendum to 'On the stability of functional equations on square-symmetric groupoid'. Nonlinear Anal. 62, 365-381 (2005)

44. Páles, Z, Volkmann, P, Luce, RD: Hyers-Ulam stability of functional equations with a square-symmetric operation. Proc. Natl. Acad. Sci. USA 95, 12772-12775 (1998)

45. Tabor, J, Tabor, J: Stability of the Cauchy functional equation in metric groupoids. Aequ. Math. 76, 92-104 (2008)

doi:10.1186/1687-1847-2013-138

Cite this article as: Batko and Brzdęk: A fixed point theorem and the Hyers-Ulam stability in Riesz spaces. Advances in Difference Equations 2013 2013:138.

\section{Submit your manuscript to a SpringerOpen ${ }^{\ominus}$ journal and benefit from:}

- Convenient online submission

- Rigorous peer review

- Immediate publication on acceptance

- Open access: articles freely available online

- High visibility within the field

- Retaining the copyright to your article 